\title{
Understanding local forces in electrophoretic ink systems: utilizing optical tweezers to explore electrophoretic display devices
}

\author{
David L. Wei ${ }^{\mathrm{a}, \mathrm{b}}$, Mark R. Dickinson ${ }^{\mathrm{a}}$, N. Smith ${ }^{\mathrm{c}}$ and Helen F. Gleeson ${ }^{\mathrm{b}}$ \\ ${ }^{a}$ School of Physics and Astronomy, and Photon Science Institute, University of Manchester, \\ Manchester, M13 9PL, UK \\ ${ }^{\mathrm{b}}$ School of Physics and Astronomy, University of Leeds, Leeds, LS2 9JT, UK \\ ${ }^{\mathrm{c}}$ Merck Chemicals Ltd.*, Advanced Technologies, Chilworth Technical Centre, University \\ Parkway, Southampton, SO16 7QD, UK \\ *Merck Chemicals Ltd is a subsidiary of Merck KGaA, Darmstadt, Germany
}

\begin{abstract}
Optical tweezers can be used as a valuable tool to characterize electrophoretic display (EPD) systems. EPDs are ubiquitous with e-readers and are becoming a commonplace technology where reflective, low-power displays are required; yet the physics of some features crucial to their operation remains poorly defined. We utilize optical tweezers as a tool to understand the motion of charged ink particles within the devices and show that the response of optically trapped electrophoretic particles can be used to characterize electric fields within these devices. This technique for mapping the force can be compared to simulations of the electric field in our devices, thus demonstrating that the electric field itself is the sole governor of the particle motion in an individual-particle regime. By studying the individual-particle response to the electric field, we can then begin to characterize particle motion in 'real' systems with many particles. Combining optical tweezing with particle tracking techniques, we can investigate deviations in many particle systems from the single-particle case.
\end{abstract}

Keywords: Optical tweezing, electrophoretic display, electrophoresis, electronic ink, electric field

\section{INTRODUCTION}

Electrophoretic displays (EPDs) are an example of a reflective, image-stable display and can be commonly found in consumer electronics such as e-readers [1]. These displays operate by moving charged ink particles via electrophoresis to form an image (Fig. 1). They are able to mimic the appearance of paper and as such are highly visible even in outdoor lighting conditions, where other displays may struggle. Such a device is highly desirable for many applications due to the extremely low power requirements. The reflective nature negates the need for a powered illumination, and the image is maintained even without power - which is only required to change the image displayed. The technology is therefore a prime candidate to replace devices such as digital signs or even traditional paper labels.

Despite the widespread availability of these devices, relatively little is understood about the physics of the underlying mechanisms, such as image stability and the particle motion itself. Optical tweezing is a versatile tool for exploring many display materials at scales that would otherwise prove problematic [2]. It has also been demonstrated that specially designed charged particles can be used to map the electric field or explore electrophoretic forces via optical tweezing [35]. They are well suited to studying EPDs, as the micron-sized electrophoretic ink particles are trappable with a standard beam profile.

In this study, the motion of micron-scale electrophoretic particles under an oscillating electric field is analyzed with optical tweezers. Standard electrophoretic ink particles are usually sub-micron size, due to the desirable high reflectivity

Optical Trapping and Optical Micromanipulation XIII, edited by Kishan Dholakia, Gabriel C. Spalding, Proc. of SPIE Vol. 9922, 99221D · ( 2016 SPIE · CCC code: 0277-786X/16/\$18 · doi: 10.1117/12.2237675 
of particles at these sizes [6]. Merck KGaA, Darmstadt, Germany provided us with particles that replicate the properties of commercially used EPD inks, yet were slightly larger in size $(1.5$ micron $)$ and therefore trappable [7]. In all experiments, the particles are dispersed in dodecane, thus accurately replicating the conditions in a standard commercial EPD. Our experiments show that the particle motion follows a linear dependency on the electric field, and we demonstrate how our technique can be extended to mapping the electric field profile in various devices. This is corroborated via electric field simulations.

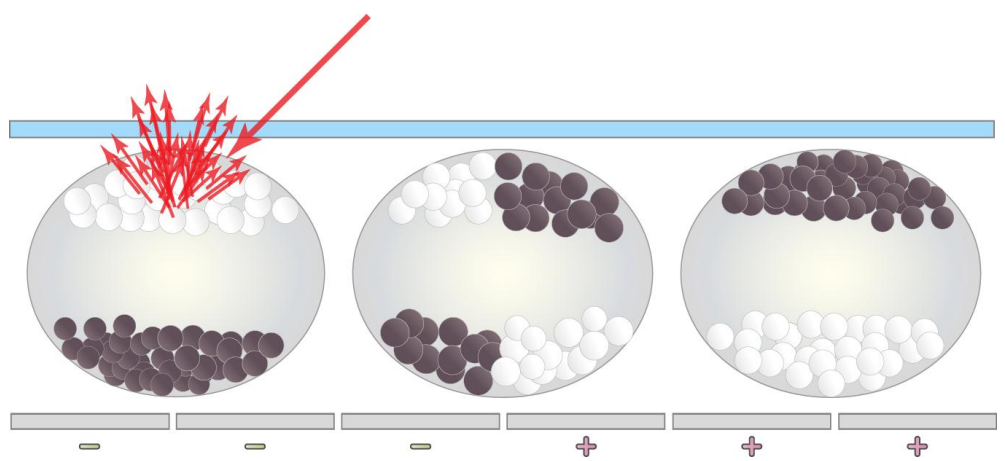

Fig. 1. Schematic showing the working mechanism of an EPD. Black, highly absorbing, ink particles with a positive charge are drawn towards negatively charged electrodes, while white, highly reflective, negatively charged particles are drawn to the opposite electrodes. This forms the basis of how an image is formed. Diffuse reflections (shown in red) from the nonuniform surface of the ink particles cause the display to appear paper-like.

\section{BACKGROUND THEORY}

The motion of electrophoretic particles under an electric field is governed by the electrophoretic mobility,

$$
\mu=\frac{\epsilon_{r} \epsilon_{0} \zeta f\left(\lambda_{D}, a\right)}{\eta}
$$

which is dependent upon the dielectric permittivity and the viscosity of the medium, $\varepsilon_{r, 0}$ and $\eta$, respectively; the zeta potential, or effective charge, of the particle, $\zeta$, and Henry's function which is dependent upon the Debye length, $\lambda_{D}$, and particle radius, $a$ [8]. While a charged particle will be accelerated under an electric field, $E$, it is assumed that the terminal velocity, $v$, is reached very quickly and thus the particle's velocity is simply characterized by [9]

$$
v=\mu E \text {. }
$$

Equations (1) and (2) can be used to determine the switching time of an EPD, an important factor governing the operation of such devices. It may be concluded from Equation (2) that using velocimetry techniques one could calculate the electric field, however, in many particle systems there will also be electrical screening and thus it is desirable to measure the field in a single-particle regime first. Measuring the velocity of individual particles in such low concentrations via velocimetry is not a simple task as the random positioning of the very few particles in the system does not allow one to be selective of the regions to be measured. Further, particle diffusion out of the plane of focus restricts the time period for which individual particle measurements can be made. However, optical tweezing allows both the selection of individual particles and their location, making it a desirable tool to use in this field.

It is well established that a particle held in an optical trap can be modelled as a linear spring [10]. This allows simple calculation of the force acting on a particle, $F_{O T}$, to be defined as

$$
F_{O T}=\kappa x
$$


where $\kappa$ is the characteristic spring constant of the trap, and $x$ is the displacement of the particle from the trap centre. The force experienced by a particle of charge, $q$, in an electric field, $E$, is also simply defined via

$$
F_{E}=q E
$$

For a particle of known charge and trap of known stiffness, it is therefore trivial to calculate the strength of the electric field by equating (3) and (4). However, in an actual electrophoretic solution other factors will affect this measurement, namely the build up of charged ions or micelles on the electrode regions, effectively screening the field. Such phenomena are more significant under direct current, so measuring the electric field in that regime should be avoided. Applying an AC field of sufficiently high frequency to an electrophoretic solution will cause any ions or charged micelles to become effectively stationary, thus avoiding build up of charge on the electrodes. Analyzing the motion of a particle in the frequency domain, by performing a Fourier transform of the particle's trajectory, allows one to pick out the amplitude of motion at a given frequency. Thus, the amplitude of motion at the driving frequency is proportional to the force applied by the electric field.

Electrophoretic (dielectric) particles are also subject to the dielectrophoretic force,

$$
F_{D E P}=2 \pi a^{3} \epsilon_{0} \epsilon_{r} \operatorname{Re}[K(\omega)] \nabla E^{2}
$$

which is dependent upon the dielectric permittivity of the medium, the real part of the frequency dependent ClaussiusMassotti function, $K(\omega)$, and the gradient of the square of the electric field. The analysis described below allows us to assess the relative contributions of the electrophoretic (Equation 4) and dielectrophoretic (Equation 5) forces on individual particles.

\section{METHODS}

\subsection{The optical tweezing setup}

The optical tweezing setup is based around an inverted microscope, with a static optical trap and a translatable, motorised stage, as shown in Fig. 2.

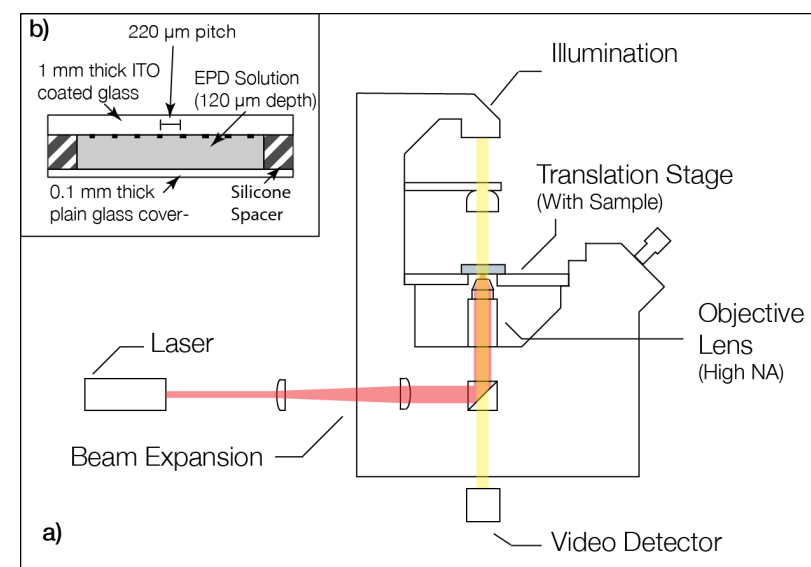

Fig. 2. a) Schematic of the optical tweezing setup used, as described in section (3.1.). b) A schematic of the cell design used to measure the response of EPD particles. The electrodes are arranged in an 'in-plane' design, meaning that they are only on one glass surface, in an inter-digited pattern, causing an electric field in the same plane as the electrodes. 
The trapping laser used is a Laser Quantum Ventus 1064, CW laser. This operates at power up to $3 \mathrm{~W}$ and at a wavelength of $1064 \mathrm{~nm}$. A series of mirrors and relay lenses are used to direct the beam into the microscope and adjust beam size to correctly fill the back aperture of the objective lens.

The objective lens used is an Olympus UPLFLN 100X oil-immersion objective lens, with a numerical aperture of 1.30, in order to tightly focus the beam into the sample. The objective lens is housed in a Leica DM-IRB inverted microscope, which also provides the necessary optics to allow imaging of the sample via digital camera.

While the optical trap itself is static, the sample can be translated by a motorised stage (Prior Proscan) allowing a full range of $x-y$ motion. The stage is driven by stepper motors, providing a spatial resolution of up to $0.04 \mu \mathrm{m}$ in both $x-y$ and axial directions.

\subsection{Imaging/tracking setup}

Imaging and live particle tracking are performed using a Prosilica EC 1280 CMOS camera, with full resolution of $1280 \mathrm{x}$ 640 pixels. This allows tracking of individual particles, when reducing the resolution to $32 \times 32$ pixels, at up to $1 \mathrm{kHz}$.

A set of LabVIEW software, previously modified in-house, and based around Glasgow's optical trapping toolkit, is used to handle live particle tracking [11]. The software outputs high resolution spatial data (order 10s nanometers), which is stored for frequency domain analysis, later on.

\subsection{Sample setup}

The electrophoretic materials used are provided by Merck KGaA, Darmstadt, Germany. They are $1.5 \mu \mathrm{m}$ diameter, polymeric particles, with charged surfactants. The particles are dispersed in dodecane, as in standard electrophoretic displays.

The $1064 \mathrm{~nm}$ wavelength trapping laser restricts the possible cell designs that can be tested. This wavelength is heavily absorbed by indium tin oxide (ITO), a common transparent conductor, and care must be taken to avoid passing the trapping beam through an ITO coated surface, as that would cause substantial local heating detrimental to the sample integrity. The measurements are therefore made using an in-plane electrode design, seen in Fig. $2 \mathrm{~b}$. This electrode pattern not only allows us to take measurements close to an electrode while avoiding local heating, but it also ensures that particle motion will be perpendicular to the beam and viewing direction, and therefore more easily resolved.

The cells used incorporate in-plane ITO electrodes of $20 \mu \mathrm{m}$ width, with inter-electrode spacing of $200 \mu \mathrm{m}$. Silicone spacers ensure a cell depth of $120 \mu \mathrm{m}$, with the opposite side of the cell being sealed with a plain glass coverslip of 100 $\mu \mathrm{m}$ thickness. The cell is filled with the electrophoretic solution, at a concentration of approximately $0.01 \%$ solids by weight.

\section{RESULTS}

\subsection{Initial measurements}

The response of the electrophoretic particles to a sinusoidal electric field, in conjunction with Fourier analysis, was used to map the electric field in the cells. 

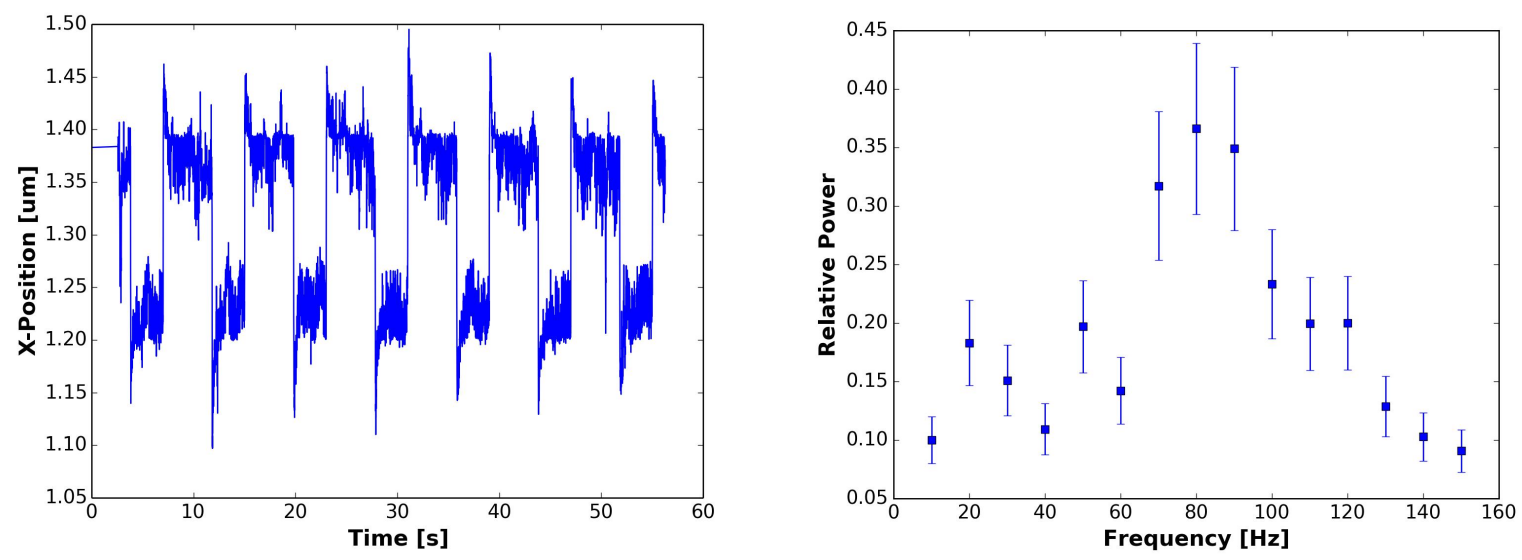

Fig. 3. Left: The motion of an EPD particle under the response of a slowly oscillating electric field with a square wave profile. Right: The relative power of an EPD particle's motion at a given driving frequency. This was used to select a driving frequency in subsequent experiments in the range $80-100 \mathrm{~Hz}$.

The displacement of the probe particle from the trap centre can be used as a direct measure of the force experienced, from equation (3). Slowly oscillating electric fields, with a square-wave profile were initially tested. This technique was capable of generating and detecting displacements of $75 \mathrm{~nm}$ from the trap centre. The impulse generated by the changing polarity of the field, seen in Fig. 3. (left), could be sufficient to displace the probe particle from the trap.

The frequency response of the particles in such a system is complicated, due to factors such as cell impedence, motion of the electrical double layer etc. therefore we aimed to constrain the working frequency. The measured power as a function of frequency for the particles in dodecane was taken over the range 5 to $150 \mathrm{~Hz}$, as shown in Figure 3. The test cell was driven over this range, while a particle was held in the trap at a fixed position. Fourier analysis was used to measure the degree of the particle's motion at the driving frequency, and allowed us to constrain the driving frequency used to between 80 and $100 \mathrm{~Hz}$.

\subsection{Electrophoretic and dielectrophoretic forces}

Analyzing the particles' motion in the frequency domain also allows us to determine whether the particle responds to the electric field either linearly, or if there are other dependencies. The presence of particle motion at a twice the driving frequency would indicate that the particle is experiencing a dielectrophoretic force in response to an electric field gradient [3]. Examining the particles' response in the frequency domain while near to an electrode (where the highest field gradients would be present), allows us to determine if any dielectrophoretic force is acting.

As described earlier (equation $4 \& 5$ ), the particles would exhibit a linear response to the electric field via the electrophoretic force, and a response to the electric field gradient via the dielectrophoretic force, which would manifest as a quadratic response i.e. peak at twice the driving frequency.

The analysis shows only a linear.response, with only the driving frequency being present in the Fourier transform. The frequency domain analysis for particles at any proximity to the electrodes did not show any harmonics of the driving frequency.

The absence of any higher order harmonics indicates that the particle is only responding to the field directly via the electrophoretic force (Equation (4)), and not as a response to field gradients via the dielectrophoretic force, Equation (5). This is as expected in dodecane, where the dielectric constant, $\varepsilon_{D}=2.0$ and therefore any dielectrophoretic force would be much lower than in a highly polar solution such as water $\left(\varepsilon_{\mathrm{w}}=80.4\right)$. While field gradients are not present in the standard design of most EPDs, this indicates that in novel device geometries, strong field gradients would not directly affect the particle motion. 


\subsection{Mapping the electric field}

The response of a particle to a set driving frequency was measured as the particle was moved through the cell both axially (away from the plane of the electrodes), and laterally (between electrodes). The relative amount of particle motion at the driving frequency was then used to quantify the force experienced by the particle from the electric field.

The experimental power measurements were normalized, and various parameterizations were fitted in order to characterize the electric field as a function of position. Regression analysis was used to assess the different parameterizations and, in both cases, it can be shown that the force experienced by the particle falls off with an inverse dependence on distance from the electrode; i.e. electric field,

$$
E(x)=\frac{a}{b-x}+c
$$

where $x$ is the distance from the electrode in microns and $a, b$ and $c$ are fitting constants, with physical interpretation as follows. The value of $a$ and $c$ indicate the maximum and minimum electric field, respectively, as a function of distance from the electrode. The parameter, $b$, indicates an offset in distance from the electrode, which can be used to correct positional data.

While the field as a function of horizontal (lateral) and vertical (axial) distance from the electrode exhibits the same characteristic fall-off, Figure 4., a subtle difference can be noticed between the cases. As one moves away from the electrode in the axial direction, the parameter $c$ showed a negative value. As this has no physical meaning, it can be interpreted as there being no minimum field in this direction. However, when moving laterally the same parameter indicates a small, positive minimum field. This would be consistent with the particle approaching another electrode as it moves in this direction.
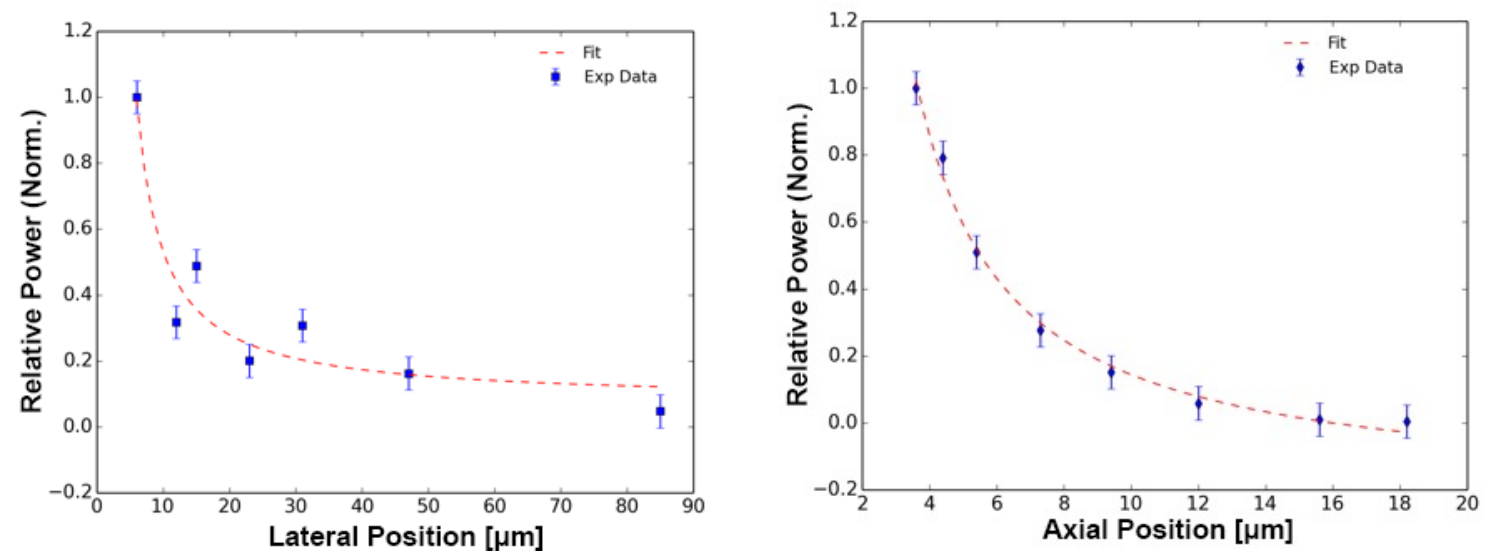

Fig. 4. The relative power measured by an oscillating EPD particle as you move laterally (between electrodes) shown on the left, and axially (away from the electrode plane), shown on the right. 


\section{ELECTRIC FIELD SIMULATION}

Simulations of the electric field were performed using in-house software, developed in MATLAB, previously described in [12]. The software computes the $2 \mathrm{D}$ electrical potential around fixed electrodes. The electric field can therefore be calculated for user specified electrode structures, device geometries, applied charges and permittivity properties.

The electric potential is defined as an input grid for the software, the resolution of which can be modified depending on the sensitivity required. Boundary conditions are applied to the grid, with mirror symmetry applied in the $x$-direction and a fixed potential applied to the $y$-direction boundaries. Electrode, or conducting, regions can be masked for calculations. The permittivity is defined as a grid intermediate to the potential grid.

The voltage applied to the electrodes is defined in the input potential grid, and the program then solves Maxwell's equations to calculate the potential throughout. A multigrid (or coarse-graining) technique is used to greatly enhance computational speed. The field is solved for a much coarser grid than is initially defined, and then iteratively refines the grid and calculates the solution until the finest resolution is reached.
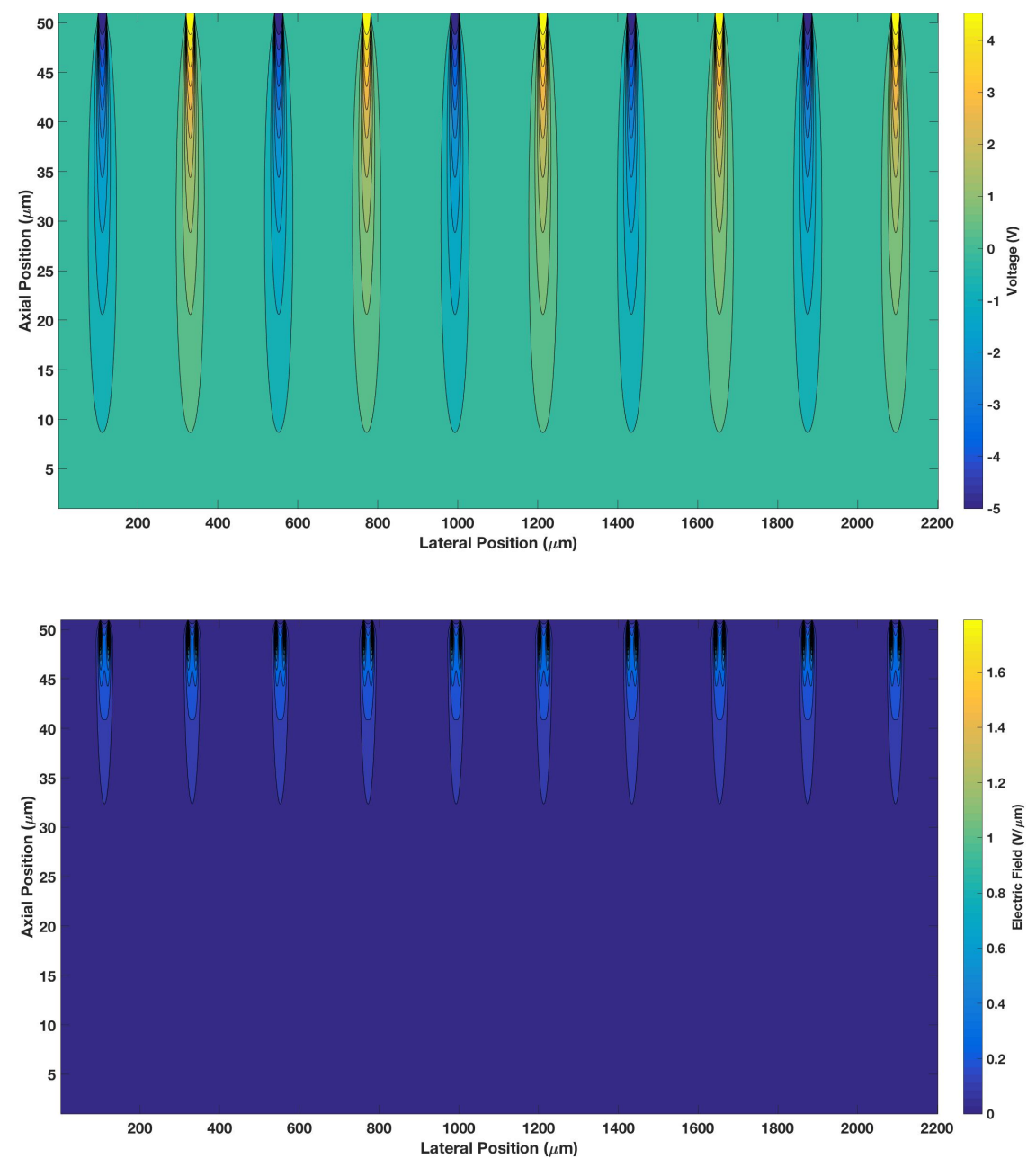

Fig. 5. Top: The voltage profile calculated via simulation for the cell described in section (3.1). Bottom: The electric field profile for the same cell, produced from the gradient of the voltage profile. 

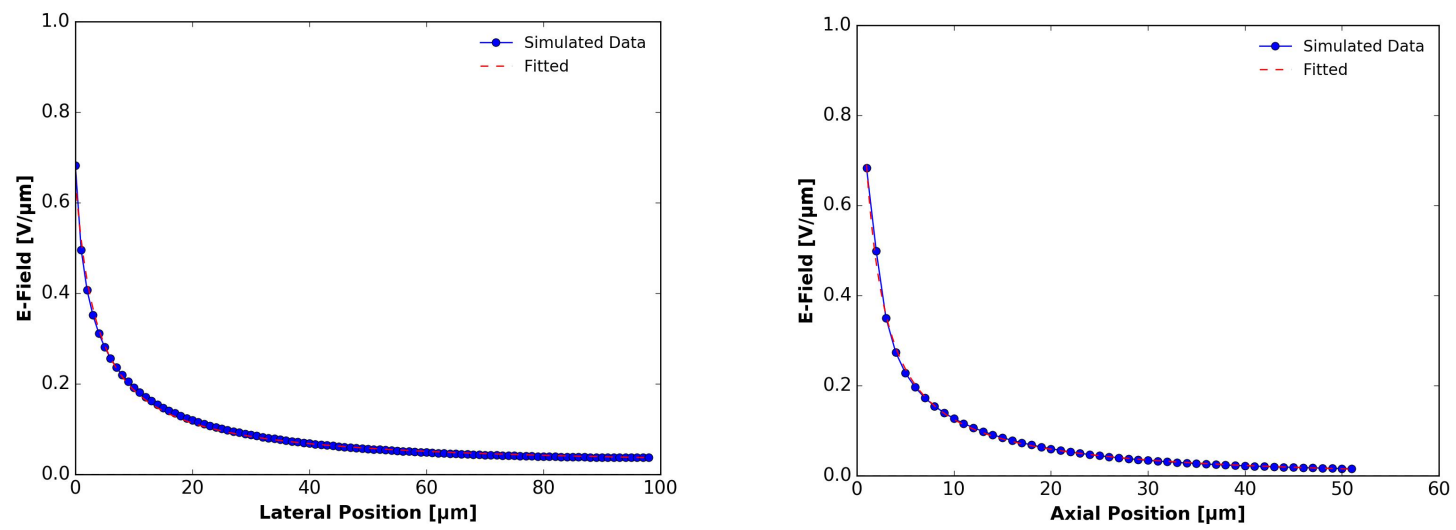

Fig. 6. Left: The simulated profile for the $\mathrm{x}$-component of the electric field, including fitted line of the form in equation (5). This was from a 1D cross-section, in the lateral plane (between electrodes). Right: As before, this time for an axial crosssection.

\subsection{Simulation results and comparison with experiment}

The simulation software was used to calculate the electric field in the experimental device described in Section (3.3), with a potential difference of $10 \mathrm{~V}$ applied across the electrodes. The 2D voltage and electric field profiles are displayed in Fig. 5. It can be seen that the areas of highest electric field are found very near to the electrodes themselves, and no strong field can be seen in either the gaps between the electrodes, or when moving in the $y$-direction away from the plane of the electrodes.

In order to compare the field deduced from simulations with that implied by the experimental work, 1D slices of the simulation were examined in both the lateral and axial planes. These slices were taken from the $x$-component of the electric field, as this was the component of motion analyzed via optical tweezing. The 1D electric field profiles were then parameterized in a similar manner to the experimental data. These profiles, with lines of fitting, can be seen in Fig. 6.

After comparing different parameterizations, the simulated electric field profiles were shown to obey the same characteristics as those of experiment, showing an inverse relationship with distance from electrode. Again, it can be seen that the field drops to zero in the axial direction, whereas in the lateral direction it falls to approximately $1 \%$ of the maximum value in mid-point between electrodes (100 $\mu \mathrm{m}$ distance).

\section{CONCLUSIONS}

Optical tweezing has been demonstrated as an effective tool when investigating electrophoretic ink particles, commonly found in EPDs. Measurements have been performed with particles and under conditions that are found in real display devices. Analysis of particle motion in the frequency domain has shown that no dielectrophoretic forces are acting on the particles in their commonly used solvent, dodecane. Moreover, this analysis technique has been extended to the mapping of the electric field within display cells. It has been shown that the optically trapped electrophoretic particles can be used to characterize the electric field as a function of position, and that it follows a simple inverse relationship with distance from the electrode. The experimental parameterization is in agreement with simulations of the electric field in a device of the same geometry. 
This work demonstrates how optical tweezing can be used to explore particle motion in actual devices, including those of novel geometries where the electric field may not be well understood. The single-particle motion explored here can now be used as a starting point when investigating many particle systems via techniques such as particle tracking.

\section{ACKNOWLEDGEMENTS}

This work was made possible by support from the Engineering and Physical Sciences Research Council, UK, and Merck Chemicals Ltd.*

*Merck Chemicals Ltd is a subsidiary of Merck KGaA, Darmstadt, Germany

\section{REFERENCES}

[1] Comiskey, B., Albert, J. D., Yoshizawa, H., and Jacobson, J., "An electrophoretic ink for all-printed reflective electronic displays," Nature 394, 253-255 (1998).

[2] Sanders, J. L., Dickinson, M. R., and Gleeson, H. F., "Laser tweezers for determining anisotropic viscosity coefficients of nematic liquid crystals." Proc. SPIE, 7762, 221-229 (2010).

[3] Pesce, G., Rusciano, G., Zito, G. et al., "Simultaneous measurements of electrophoretic and dielectrophoretic forces using optical tweezers", Optics Express 23(7), 9363-9368 (2015).

[4] Otto, O., Gutsche, C., Kremer, F. et al. "Optical tweezers with $2.5 \mathrm{kHz}$ bandwidth video detection for singlecolloid electrophoresis.” Rev. Sci. Inst. 79, 023710 (2008)

[5] Semenov, I., Otto, O., Stober, G. et al. "Single colloid electrophoresis", J. Colloid and Interface Sci. 337, $260-$ 264 (2009)

[6] Fitzhenry-Ritz, B. "Optical properties of electrophoretic image displays", IEEE Trans. Electron Devices 28(6), 726-735 (1981)

[7] Goulding, M., Smith, N., Farrand, L. et al. "23.1: Invited paper: colloidal dispersion materials for electrophoretic displays and beyond.”, SID Symp. Digest of Tech. Papers 46 (1), 326-329 (2015).

[8] Hirst, L. S., [Fundamentals of Soft Matter Science], CRC Press (2012)

[9] Amundson, K., Eds: Chen, J., Cranton, W., and Fihn, M., [Handbook of Visual Display Technology] Springer, 1700-1712 (2012)

[10] Neuman, K. C., Nagy, A., "Single-molecule force spectroscopy: optical tweezers, magnetic tweezers and atomic force microscopy." Nature Methods 5(6), 491-505 (2008).

[11] Gibson, G. M., Leach, J., Keen, S., Wright, A. J., Padgett, M.J. "Measuring the accuracy of particle position and force in optical tweezers using high-speed video microscopy" Optics Express 16(19), 14561-14570 (2008).

[12] Milton, H., Brimicombe, P., Morgan, P., Gleeson, H. F., \& Clamp, J., 'Optimization of refractive LC lenses using an efficient multigrid simulation’, Optics Express, 20 11159-11165 (2012). 